\title{
Médiévales
}

Langues, Textes, Histoire

\section{Réflexions sur les objets et les pratiques de l'histoire de l'art médiéval. À propos de L'objet de l'histoire de l'art, de Roland Recht}

\section{Daniel Russo}

\section{(2) OpenEdition \\ Journals}

Édition électronique

URL : https://journals.openedition.org/medievales/1463

DOI : 10.4000/medievales. 1463

ISSN : 1777-5892

Éditeur

Presses universitaires de Vincennes

Édition imprimée

Date de publication : 1 décembre 2006

Pagination : 139-152

ISBN : 978-2-84292-193-4

ISSN : 0751-2708

Référence électronique

Daniel Russo, «Réflexions sur les objets et les pratiques de l'histoire de l'art médiéval. À propos de L'objet de l'histoire de l'art, de Roland Recht ", Médiévales [En ligne], 51 | automne 2006, mis en ligne le 27 mars 2009, consulté le 24 avril 2022. URL : http://journals.openedition.org/medievales/1463 ; DOI : https://doi.org/10.4000/medievales. 1463

Ce document a été généré automatiquement le 24 avril 2022.

Tous droits réservés 


\section{Réflexions sur les objets et les pratiques de l'histoire de l'art médiéval. À propos de L'objet de l'histoire de l'art, de Roland Recht}

Daniel Russo

\section{NOTE DE L'ÉDITEUR}

Roland Recht, L'objet de l'histoire de l'art, Paris, 2003 (Leçons inaugurales du Collège de France). Cet article a été précédé par une étude plus générale d'historiographie, présentée à l'Université de São Paulo en septembre 2003 et, depuis, publiée sous le titre : « Histoire de l'Art médiéval et Iconographie : Tendances actuelles de la recherche ", Le Moyen Âge vu d'ailleurs II. Historiografia e Pesquisas Recentes, E. MAGNANI, H. Franco JunIOR, Fl. DE CAMPos dir., Estudos Avançados da Universidade de São Paulo 27, 2004, p. $110-123$.

Il y a à peine plus de trente ans, dans le volume collectif dirigé par Jacques Le Goff et Pierre Nora, sous l'intitulé Faire de l'histoire. Nouvelles approches, Henri Zerner traçait un bilan des recherches en histoire de l'art et en iconographie assez pessimiste, en ces termes: «... L'histoire de l'art, le discours sur l'art, se trouve pris, pour ne pas dire coincé, entre l'histoire et la critique. Empirique et positiviste, l'histoire de l'art traditionnelle se montre extrêmement méfiante à l'égard de toute théorie et même de toute interprétation approfondie des œuvres. La critique, de son côté, prend presque toujours pour postulat ce qu'elle cherche à cerner, à définir, à éclairer dans l'œuvre, ce qui fait qu'elle est œuvre d'art, échappe au temps et par conséquent à l'histoire. On a pourtant affirmé, je serais tenté de dire démontré, qu'une réflexion bien fondée sur l'art, une science de l'art, ne pouvait qu'être à la fois historique et théorique ${ }^{1}$. » Il citait Erwin Panofsky (1892-1968) et un article rédigé en $1925^{2}$, et reconnaissait que «[...] 
l'histoire de l'art, qui souffre depuis un demi-siècle d'une profonde stagnation théorique, n'est pas en état de répondre aux questions qui lui sont posées ${ }^{3}$.»

2 Dans sa conférence donnée à l'Université de tous les savoirs, le 7 avril 2000, Hans Belting dressait un bilan qui n'était guère plus optimiste et avouait aussi les difficultés d'une discipline qui, d'une part, refusait de se penser elle-même, d'autre part, ne se reconnaissait pas dans le questionnement de l'histoire. Il écrivait notamment : « ... Loin d'être un modèle universel, l'histoire de l'art telle qu'elle se pratique habituellement ne constitue donc qu'une figure possible du récit historique. Pour peu qu'on y réfléchisse de plus près, le problème d'une science de l'art aujourd'hui révèle sa véritable dimension. Croire que seule l'idée d'histoire se serait modifiée tandis que l'idée d'art serait restée la même est absolument faux. L'idée d'art est en fait intimement liée au processus historique qui s'est déroulé dans la modernité ; elle est donc elle-même un produit de l'histoire ${ }^{4} . . . »$

3 C'est à la suite de ces interrogations que Roland Recht situe, d'emblée, le problème dont il entend traiter au cours de sa leçon inaugurale du Collège de France, le 14 mars 2002 : «L'expression histoire de l'art n'assigne-t-elle pas clairement à ce genre historial son complément - autrement dit l'art sous les multiples formes visuelles qu'il revêt - sans qu'il soit nécessaire d'en repérer un autre ? En d'autres termes encore : est-ce l'histoire qui est l'objet de notre recherche, ou l'art 5 ? » La question a de quoi surprendre sous la plume d'un historien de l'art qui écrit et enseigne dans le champ de la discipline et qui œuvre tant pour sa reconnaissance. Elle est à insérer, pourtant, dans l'ensemble des réflexions qui l'ont précédée. Qu'en est-il vraiment aujourd'hui? Les remarques avancées par Roland Recht permettent d'examiner la situation dans le long terme chronologique, autour de quelques propositions qui orientent le débat tout en l'illustrant sur certains points, par exemple celui du style gothique ${ }^{6}$. Stimulant et fécond, ce petit livre dense invite à reconsidérer la place occupée par l'histoire de l'art dans l'Université, puis ses problématiques d'étude, dans ses rapports à l'histoire et à l'interprétation des œuvres.

Situations

4 Par comparaison avec les lettres, les langues, la philosophie et l'histoire, l'histoire de l'art s'est affirmée, de manière récente, comme une discipline dans le système universitaire français. Au sein du Collège de France, elle est "plus ou moins directement présente depuis 1878. C'est, en effet, une chaire d'esthétique et d'histoire de l'art qui sera créée pour Charles Blanc [...], puis pour son successeur Henri Guillaume $[\ldots]$ : cette situation témoigne d'une perception encore floue d'une discipline qui, en France du moins, n'avait pas encore un statut bien défini. ${ }^{7}{ }^{1}$ 'histoire de l'art devait délimiter son champ et ses approches face à l'esthétique, "largement imprégnée d'hégélianisme ", et à l'archéologie. Dans l'Université, la première chaire d'histoire de l'art, en art moderne, date de 1899, et est ouverte à la Sorbonne dans le cadre général d'un enseignement de l'histoire de la civilisation à l'époque moderne : elle fut attribuée à Henry Lemonnier qui venait de publier, en 1893, ses Études d'art et d'histoire. L'art français au temps de Richelieu et de Mazarin, en s'intéressant à "replacer l'art dans l'histoire » et, pour l'architecture et la peinture, à voir «si vraiment elles ont quelques rapports avec l'histoire ambiante ${ }^{8}$. Henry Lemonnier avait fait une thèse en histoire $\mathrm{du}$ droit antique, sur la condition des affranchis durant les trois premiers siècles de l'Empire romain (1887) et établi une distinction nette entre des faits d'histoire politique et des faits de culture. Même si l'art lui paraissait devoir être situé dans l'histoire, il 
était étudié à un niveau très général: en effet, seule comptait l'appréciation des qualités esthétiques qui n'entraient pour rien dans le schéma de compréhension historique. Dans un article paru en 1895, sur l'art et le goût de l'époque classique en France, Henry Lemonnier écrivait que : «... C'est seulement chez les artistes médiocres que nous retrouvons les principes dans leur pure application, c'est-à-dire chez ceux qui ont pensé les leçons des maîtres, qui ont pris au pied de la lettre les théories proclamées si solennellement. Elles nous apparaissent alors dans tout le vide de leur conception ou la banalité de leur inspiration ${ }^{9}$ ". L'histoire restait un support chronologique commode, sans plus.

5 Ce genre d'interprétation remonte au fondement même de l'intérêt porté aux œuvres d'art. Roland Recht met ainsi en valeur deux attitudes opposées, l'une caractérisant plutôt l'Europe méditerranéenne et, au premier rang, l'Italie, l'autre marquant en profondeur l'Europe septentrionale. En Italie, il repère justement une conception reposant sur la continuité toujours perceptible entre le monde classique et le monde moderne, en conséquence la volonté très claire d'insérer l'art dans la mémoire collective de la cité et au milieu des liens politiques et historiques de la communauté ${ }^{10}$. À Florence, par exemple, Leone Battista Alberti (1404-1472) esquisse dans le De re aedificatoria une interprétation neuve de l'œuvre d'art en contexte civique en donnant toute sa pertinence à la réalisation achevée, plutôt qu'à la figure de l'artiste ou à celle du commanditaire ${ }^{11}$. Dans l'Europe du nord, l'absence de continuité est, en revanche, évidente: très tôt, un conflit surgit, de nature quasi-épistémologique, qui opposa l'«antiquaire ", s'occupant des objets, à l'historien, cherchant le sens et l'interprétation des œuvres. Entre la fin du XVII ${ }^{\mathrm{e}}$ siècle et celle du XIX ${ }^{\mathrm{e}}$, le statut des traces matérielles du passé évoluait aussi, en passant du document non linguistique, dont il s'agissait de reconnaître l'authenticité, au monument pour lequel jouait la compréhension historique ${ }^{12}$. Par le même mouvement, la période du Moyen Âge regagnait en intérêt par rapport à l'Antiquité et au monde moderne. Alors que l'antiquaire tendait, à l'égal du collectionneur, à réunir des objets pour produire un inventaire descriptif jamais clos, par définition, l'historien de l'art se tournait vers la connaissance des civilisations, dont l'art faisait partie selon lui ${ }^{13}$.

Deux attitudes étaient désormais bien campées et devaient perdurer même si, autour des années 1900, la synthèse semblait possible ${ }^{14}$. Pour partie, Émile Mâle (1862-1954) la réalisa dans ses grands ouvrages sur l'art du Moyen Âge et les réformes religieuses ${ }^{15}$. Il introduisit l'art dans l'histoire, tout en se considérant comme historien et archéologue, d'après les termes qu'il utilisait le plus souvent. Dans ses travaux, il situait l'histoire de l'art dans la discipline historique. Sur la sculpture médiévale, il notait: «... Les considérations esthétiques que la sculpture romane pourrait inspirer à un homme de goût, mais étranger aux études archéologiques, seraient donc de peu de valeur. Un tel art ne s'explique guère que par l'histoire ${ }^{16}$. » Dans sa thèse sur L'art religieux $d u$ XIII siècle en France, il observait: " ... L'art religieux du XIII" siècle est une écriture sacrée dont tout artiste doit apprendre les éléments $"{ }^{17}$. Il inscrivait, cependant, l'histoire de l'art dans une quête de la signification. Dans L'art religieux de la fin du Moyen Âge en France, paru en 1908, il pointait la répétition des figures des saints sur les portails des églises et s'étonnait du peu d'intérêt suscité par ces récurrences qui, à ses yeux pourtant, faisaient sens ${ }^{18}$. À partir de l'objet sculpté, qui est aussi une œuvre d'art, et non plus du désir d'inventaire ou d'un système donné a priori, la tâche consistait, dès lors, dans le fait de comprendre les différentes étapes de la fabrication et de la 
destination, afin de préciser le sens possible. Le questionnement est neuf, original, à l'écart d'autres voies ouvertes en même temps, par exemple par Henri Focillon (1881-1943), en suivant d'autres principes et d'autres exigences, portant sur des questions de style et de forme, dans des ensembles finis ${ }^{19}$. Dans ce dernier cas, à l'interrogation sur le sens on substituait « le présupposé d'une filiation dans laquelle l'œuvre singulière devrait nécessairement s'insérer» et "le présupposé d'une évolution linéaire vers une plus grande complexité, plaçant toujours les formes les plus simples à l'origine de formes plus élaborées " ${ }^{20}$. Riche d'aperçus et d'apports, cette voie d'approche synthétique, que nous retrouverions dans certains travaux d'André Chastel (1912-1990), a laissé place à une conception analytique de l'objet et du style, détachée tout autant des seules préoccupations de contenu.

7 L'histoire de l'art est ainsi parvenue à un tournant dans la définition de ses grandes orientations de recherche, au moment où elle a obtenu, avec la création de l'Institut national d'histoire de l'art, une reconnaissance politique, cela au moment où elle est enseignée à l'école, même de manière discutable. Au sein de l'Université, en revanche, sa situation apparaît nettement plus contrastée. La discipline demeure encore largement introuvable, logée souvent, et mal, par des disciplines voisines qui l'annexent, comme l'histoire, les langues et civilisations, les langues anciennes ou, encore, la philosophie, au titre de l'esthétique, et la littérature, à celui de la critique artistique, quand ce n'est pas au titre tout simple de la critique journalistique. La concurrence s'avère plus difficile du côté des arts plastiques, qui se sont éloignés de l'étude des seuls "arts vivants ", à leur origine pourtant, et qui possèdent une façade institutionnelle irréprochable avec la préparation aux concours de recrutement, capes et agrégation. Que dire des départements de sociologie et de psychologie qui s'intéressent, de plus en plus, aux rapports entretenus par les sociétés et les arts ? ${ }^{21}$. Face à ces difficultés, et dans un pays comme la France où les recherches académiques ont plutôt défendu le savoir des "experts » contre l'interprétation des "autres », en reproduisant la vieille distinction entre les artistes, les amateurs, les profanes, il ne peut exister de réponse structurée, ni scientifiquement fondée, sur une théorie de l'art et des pratiques renouvelées en profondeur ${ }^{22}$. Selon ces perspectives, l'histoire de l'art resterait un chantier ouvert.

L'inventaire des possibles

8 Il est vrai que la difficulté est grande et semble quasiment insurmontable. Comment, en effet, interpréter le visible par un discours écrit ? Comment passer de l'un à l'autre dans une interprétation globale ${ }^{23}$ ? À la base de tout le travail, quelles que soient les orientations choisies, demeure la nécessité de constituer la documentation réunie en matériau d'étude: de là viennent les récolements d'objets, l'établissement des biographies d'artistes ou des parcours d'ateliers, les nécessaires attributions et datations. Dans ce champ des pratiques prévaut la spécificité du catalogage, donc de l'exposition et du musée, comme l'occasion et le lieu naturel à ce genre d'opération. Il est intéressant de comparer, une fois de plus, des attitudes et des réflexions distantes d'un peu plus d'une trentaine d'années, à partir des grandes manifestations qui avaient marqué les esprits des contemporains, pas toujours du reste en histoire de l'art médiéval. En 1972, au début du catalogue de l'exposition sur Georges de la Tour, Jacques Thuillier remarquait: «... La Tour est le triomphe de l'histoire de l'art, et sa justification ${ }^{24}$. " La formule sonnait juste, tant elle consacrait l'ensemble des tâches, souvent les plus minutieuses et les plus érudites, pour reconstituer un nom, une œuvre, une signature d'artiste, dans l'environnement social et culturel du XVII ${ }^{e}$ siècle. En 2003, 
s'essayant à répondre à la délicate question « Qu'est-ce que l'art? », Jacques Thuillier affirmait que « ... l'art consist(ait) en formes inédites créées par l'homme » ${ }^{25}$. L'art se retrouve isolé à l'intérieur d'une spécificité supposée, soumis à un questionnement élaboré de manière autonome, sans liens avec le processus historique. L'histoire des styles, sous sa forme la plus pure, avait, de même, proscrit de son explication les facteurs qui n'étaient pas " originellement artistiques $"{ }^{26}$. Dans le domaine médiéval, depuis vingt-cinq ans bientôt, ce sont de grandes expositions qui rythment l'avancée des connaissances sur telle ou telle période, circonscrite avec soin, étudiée à travers ses objets méthodiquement classés, depuis les Fastes du gothique (1981) jusqu'aux Princes des fleurs de lys et l'art des années 1400 (2004), en passant par L'art au temps des rois maudits (1998). Chacune de ces expositions a donné lieu à un grand moment d'érudition partagée qui visait à reconnaître, au-delà du style et de la technique, les attributions les plus récentes et à proposer de nouvelles hypothèses sur les dossiers ouverts. Mais, dans ces assemblages d'objets et à travers les pages de catalogues abondants, ne sont jamais énoncés les fondements d'un discours analytique ni ceux d'une interprétation critique. De même, des travaux remarquables sont proposés autour des livres à peintures, en 1993, dans l'exposition sur les Manuscrits à peintures en France (1440-1530), en 2003, dans celle sur Jean Fouquet. Peintre et enlumineur $d u \mathrm{xv}^{e}$ siècle, toutes deux à la Bibliothèque nationale de France (site Richelieu) et coordonnées par François Avril, avec la collaboration de Nicole Reynaud pour la première. Or, l'ensemble de ces très belles réalisations reste, malgré tout, dominé par la conception d'une histoire qui sert de cadre chronologique commode, tout tracé, pour regrouper des objets d'art, des livres et des œuvres précieuses, qui relèvent de la même conjoncture. En dépit de toutes les informations rendues disponibles, de tous les classements effectués et de toutes les chronologies établies, aucun discours scientifique sur l'art du Moyen Âge n'a pu être fondé, aucune hypothèse d'envergure sur le statut même de ces objets si précieux. À la fin $d u$ xix siècle et au tout début $d u x^{e}$, à Vienne, s'occupant du musée des arts appliqués, Alois Riegl (1858-1905) avait esquissé une interprétation générale de ces arts dits, à tort, "mineurs ", en faisant la synthèse des objets dont il avait la garde ${ }^{27}$. De manière plus restreinte, mais susceptible de résultats plus directs, Roland Recht propose de recentrer l'analyse sur l'objet, et non sur «le système ", en précisant les principaux agents qui interviennent dans sa réalisation: le commanditaire; le modèle suivi, explicitement ou implicitement; la subjectivité de l'artiste ${ }^{28}$. Les deux premiers agents peuvent déterminer telle ou telle formulation particulière au cours d'un processus sélectif qui aboutit, en fin de compte, à l'objet réalisé. Le troisième agent est plus délicat à distinguer, compte tenu des genres de sources disponibles et des comportements les plus habituels par rapport aux artistes ${ }^{29}$.

9 Le choix des formes serait la réponse à une demande initiale, si bien que ces formes ne sont pas une donnée absolue mais relative. En effet, des œuvres ou des objets destinés à des commanditaires, se trouvant en des lieux différents ou présentant des profils sociaux distincts, peuvent être traités suivant un répertoire de formes très différentes, d'autant plus qu'elles répondent à des situations et à des fonctions particulières. Par exemple, au XIII ${ }^{\mathrm{e}}$ siècle, les sculpteurs allemands pratiquaient un genre humble, bien éloigné du grand style des portails français, mais avaient pu (et su) travailler aussi dans ce style à Reims ${ }^{30}$. Sans exagérer la détermination des genres de discours sur l'emploi de tel ou tel style de commande, car tout dépend de la manière dont on les envisageait à l'époque, force est de reconnaître qu'ils définissent des cadres commodes pour la reconnaissance du statut des œuvres et de «la forme du sens ", c'est-à-dire du style ${ }^{31}$. 
Plus largement, la question de la demande doit être replacée à l'intérieur d'un ensemble plus vaste, que certains n'hésitent pas à qualifier de marché des biens de consommation ${ }^{32}$ en distinguant ainsi le niveau économique de la commande, puis la mise en œuvre d'un modèle de consommation, religieux dans une grande mesure et adapté aux fonctions liturgiques, enfin l'émergence relative, au cours du $\mathrm{xv}^{\mathrm{e}}$ siècle, de nouvelles modes et de nouveaux objets ${ }^{33}$. Par ce biais, l'étude des " patrons ", cartons et dessins, fait pénétrer davantage dans la réalisation même de tous ces objets, d'un triple point de vue économique, social et culturel, et fournit la base d'observations nécessaire à une réévaluation raisonnée des rapports entre les artistes et les commanditaires ${ }^{34}$.

10 Les structures économiques et sociales de la demande ne sauraient pourtant former l'horizon indépassable de la recherche. S'ajoutent aussi la politique de l'objet et les désirs attachés à sa possession. C'est ce qui ressort le mieux des grandes expositions célébrant l'art princier des fleurs de lys, autour des années 1400 : à Paris comme à Bourges ou en Bourgogne, les princes s'affairaient pour voir réaliser les plus beaux, les plus précieux, des ivoires, des reliquaires et des émaux; ils dépensaient des trésors pour arriver à leurs fins et manifester, de la sorte, leur pouvoir et leur magnificence ${ }^{35}$. Impliqués dans un jeu d'échanges complexes, où interféraient constamment les liens de l'argent et de la politique, ces objets-images tissaient une mémoire lignagère et un dense écheveau de relations inter-générationnelles, transmis souvent par les femmes ${ }^{36}$. Dès lors, un statut juridique émerge, peu à peu, pour ces réalisations de prix, que les textes essaient de codifier à partir de l'intérêt porté aux matériaux, dont elles se composent, et à leurs différentes modalités d'agencement ${ }^{37}$.

11 La question de la demande artistique, pour la période médiévale, figure bien au nombre des préoccupations essentielles à la définition d'une histoire de l'art consciente de ses responsabilités en tant que discipline scientifique.

L'interprétation ou le sens des œuvres

Désireux d'assurer la scientificité de son approche, en la situant dans le temps historique, Alois Riegl avait utilisé, pour répondre à la question de la demande, le terme de kunstwollen, difficilement traduisible en français, qu'on peut rendre par volonté, vouloir au sens générique, intention d'art ${ }^{38}$. En recourant au terme de wollen et non à celui de wille (volonté individuelle), Riegl entendait désigner un ensemble de réalités psychologiques qui ne dépendaient pas seulement de la personne individuelle, mais la dépassaient sous la forme d'une tendance collective ${ }^{39}$. Le terme désignait, d'autre part, tantôt une pulsion non dominée par la conscience, tantôt une activité plus délibérée et plus consciente de ses fins. Enfin, si le kunstwollen se manifestait chez l'artiste comme une manière de représenter les choses en accentuant certains de leurs aspects et en en réprimant d'autres, il se donnait dans le public comme un besoin corrélatif de voir les choses ainsi représentées ${ }^{40}$. Par conséquent, l'aire sémantique du terme a évolué dans la pensée de Riegl et varié dans un même discours. Riegl parlait donc du kunstwollen d'un peuple, de celui d'une époque ou d'un artiste, enfin de celui des commanditaires. Ce qui rend aujourd'hui plus encore ambiguë l'utilisation de la notion, voire contradictoire, devait paraître un avantage dans la pratique de Riegl qui, traitant avec souplesse les divers sens du terme sans avoir à rendre compte de leur articulation, pouvait passer sans heurts du style d'une époque au style d'un artiste, comme de celuici ensuite au style reçu en partage par tout un public, selon sa conception de l'histoire qui privilégiait la continuité sur la rupture. Une autre ambiguïté subsiste, portant non 
sur le contenu, mais sur le statut épistémologique du concept. Dans la Spätrömische Kunstindustrie, Riegl le présentait comme une conception téléologique; dans « Naturwerk und Kunstwerk », comme le seul donné certain de l'histoire de l'art, tandis que ce qui le détermine ne peut que faire l'objet de suppositions métaphysiques. Il demeurait possible d'éclairer la nature du kunstwollen d'une époque en le comparant à l'orientation des autres domaines de la culture du même peuple : religion, philosophie, droit. Selon Riegl, cela permettait de constater que le Kunstwollen n'était pas déterminé par ces autres domaines, mais que tous montraient des développements parallèles issus d'une même attitude fondamentale de l'homme, inséré dans son époque, vis-à-vis du monde ${ }^{41}$. Il visait ainsi à trouver, dans cette signification, le point d'intégration de l'art et de la culture et, en particulier, de l'iconographie et de la forme, tout en préservant la spécificité de l'art, qu'il voyait dans la réalisation formelle et dont il cherchait à caractériser ce qu'on appellerait aujourd'hui la structure. Pourtant, sa conception a fait l'objet de vives critiques, parce qu'elle détournait de l'étude des relations historiques concrètes entre tous les domaines de la culture auxquels participait l'artiste ${ }^{42}$. Pour Riegl, toutefois, il s'agissait par ce biais de considérer la création artistique elle-même, telle qu'on pouvait la saisir par une appréhension globale de l'œuvre dans l'organisation particulière des divers éléments qui constituaient la forme. Par là, il voulait fonder une histoire de l'art scientifique sur la spécificité de l'art ${ }^{43}$.

Dans la pensée critique allemande du début du $\mathrm{xx}^{\mathrm{e}}$ siècle, le concept de kunstwollen a connu deux interprétations divergentes. D'une part, pour Edgar Wind (1900-1971) et Hans Sedlmayr, c'était une force réelle qui agissait dans l'histoire, une volonté supraindividuelle, objective, qui se manifestait dans l'activité de groupes de personnes, sans doute en suivant la pensée de Riegl au plus près ${ }^{44}$. D'autre part, pour Erwin Panofsky, le kunstwollen, compris en tant qu'une donnée de la psychologie de l'artiste ou de la psychologie collective propre à une époque, serait inconnaissable. De plus, une approche empirique fondée sur l'aperception qu'en aurait le spectateur ne livrerait d'informations que sur la psychologie de ce dernier. La notion était donc à utiliser comme un sens immanent que la critique, au terme de sa lecture des œuvres, déchiffrait a posteriori dans les faits artistiques ${ }^{45}$. Dès lors, le sentiment de nécessité, que l'historien de l'art croyait reconnaître dans le développement d'un style, n'était plus le fait d'une téléologie menant à une essence absolue à partir de la succession des œuvres, mais l'expression de la cohérence du sens, que l'historien avait su en dégager. Pour Panofsky, et c'était là son apport personnel, loin d'avoir un caractère psychologique, le sens devait être transcendantal, saisir l'art dans son essence particulière et non en fonction des facteurs extérieurs: circonstances historiques, présupposés psychologiques, analogies stylistiques. Ainsi posé, le kunstwollen était défini à partir des concepts fondamentaux a priori, qui se référaient «non pas au phénomène lui-même mais aux conditions de son être-là et de son être tel ». Riegl n'avait jamais opéré de semblable distinction entre les réalités historiques et le sens qu'y déchiffrait l'historien. Au contraire, Panofsky replaçait la demande artistique à l'entrecroisement de ses deux champs d'observations, celui des œuvres, celui des faits historiques, sans plus supposer de continuité entre les deux, mais seulement des passages qualitatifs : dans le premier, l'étude des thématisations en cours; dans le second, celle des configurations sociales et culturelles à l'intérieur desquelles s'inséraient les thèmes et les motifs artistiques qui venaient d'être élaborés. Les notions de thèmes et de motifs étaient susceptibles de prolongements très féconds pour l'analyse des différents niveaux de signification à l'œuvre dans les arts visuels, en 
peinture, sculpture et architecture. Autour d'un noyau figuratif stable, qu'il voyait comme un signifiant, Panofsky décrivait un motif ou un ensemble de motifs, soumis à des variations incessantes, de forme et de sens. En s'appuyant sur les deux premiers niveaux préalables de l'analyse, ce qu'il nommait le "pré-iconographique » et l'« iconographique », il parvenait à distinguer la conception de l'œuvre, c'est-à-dire « la signification intrinsèque " ou encore "l'ultime contenu", selon ses expressions. Il traduisait ce qu'Ernst Cassirer (1874-1945) avait désigné par l'expression de "l'ordre symbolique $"{ }^{46}$. Il ne s'agissait pas, à ce degré de conceptualisation, de mise en contexte socio-culturel ou de vagues conditions de production de l'œuvre d'art, mais plutôt du contenu de l'art, qui était «à la mesure de l'essence » ou, suivant ce qu'en dirait Karl Manheim, du «sens de l'essence » toujours lié à l'œuvre réalisée intrinsèquement, repérable dans ses composantes, matérielles et formelles, en étroit rapport avec le signifiant général, que donnaient les deux premiers niveaux de lecture. À la manière toute contemporaine des folkloristes, tel Arnold van Gennep (1873-1957), Erwin Panofsky construisait une sémantique générale, mais appliquée à l'œuvre d'art ${ }^{47}$. Sur cette voie, il ne fut guère suivi. Lui-même, du reste, s'en éloigna, privilégiant un autre genre d'approche plus orientée vers le déchiffrement des œuvres suivant un plan de symbolique générale ${ }^{48}$. Pourtant, dans l'explication d'ensemble qu'il avait tentée de l'art, Erwin Panofsky était parvenu à réunir, très étroitement, l'histoire de l'œuvre dans sa forme comme dans ses significations, en vue d'établir un savoir sur l'art.

Après lui, les problèmes furent envisagés différemment en perdant, peut-être, le sens de cette unité nécessaire, mais en la retrouvant à l'intérieur même du champ historique. En concluant sa leçon inaugurale, Roland Recht notait «[...] la place qui reven[ait] à toutes les formes de procédures discursives » élaborées pour expliquer ce savoir sur l'art, c'est-à-dire pour produire une somme d'interprétations possibles ${ }^{49}$. $C^{\prime}$ 'est à ces conditions, d'ouverture et de réflexion, que se dessinent les principales lignes d'un ressourcement de l'histoire de l'art au sein des autres sciences humaines.

\section{NOTES}

1. H. ZERNER, « L'art », dans J. LE GOFF et P. NORA dir., Faire de l'histoire, t. 2, Paris, 1974, p. 183.

2. E. PANOFSKY, « La réflexion sur l'art, après la déroute des systèmes esthétiques », repris dans Les sciences humaines et l'œuvre d'art, Bruxelles, 1969, p. 7-47.

3. H. ZERNER, loc. cit., p. 183.

4. H. BeltiNG, «L'histoire de l'art au tournant », Université de tous les savoirs, t. 20, L'art et la culture, Paris, 2002, p. 23-24. ID., Das Ende der Kunstgeschichte?, Munich, 1983 (trad. fr. par J.-F. Poirier et Y. Michaud, Nîmes, 1989).

5. R. RECHT, op. cit., p. 16.

6. R. RECHT, op. cit., p. 43-54. ID., Le croire et le voir. L'art des cathédrales (XII ${ }^{e}-X V^{e}$ siècle), Paris, 1999 ; ID., « Wilhelm Vöge, Louis Grodecki et la première sculpture gothique ", dans W. ScHLINK dir., Wilhelm Vöge und Frankreich, Fribourg, 2004, p. 201-216. 
7. ID., op. cit., p. 12.

8. Sur l'évolution des chaires d'histoire de l'art à l'Université, L. THERRIEN, L'histoire de l'art en France. Genèse d'une discipline universitaire, Paris, 1998, p. 314-332. Les citations de H. Lemonnier sont respectivement aux p. VI et 12 de ses Études d'art et d'histoire.

9. H. LEMONNIER, « Les origines de l'art classique en France au XVI ${ }^{\mathrm{e}}$ siècle », Revue internationale de l'enseignement 1, 1895, p. 139-155 ; repris dans ID., L'art moderne (1500-1800). Essais et esquisses, Paris, 1912, p. 104.

10. R. RECHT, op. cit., p. 18-19.

11. Alberti se consacre à la rédaction du traité à partir de 1444 et en poursuit la tâche jusqu'à sa mort. L'ouvrage est imprimé en 1485. Leone Battista Alberti, L'art d'édifier, trad. fr. F. Choay dir., Paris, 2004. Voir F. FuRlan, Studia Albertiana. Lectures et lecteurs de L. B. Alberti, Turin-Paris, 2003, sur l'importance de la communauté civique dans la genèse, puis la diffusion, de l'œuvre ; F. MARIANI ZINI éd., Penser entre les lignes. Philologie et philosophie au Quattrocento, Presses universitaires du Septentrion, 2001.

12. R. RECHT, op. cit., p. 19-20 ; p. 21-22.

13. L'évolution se produit de façon concomitante en archéologie et aboutit à une semblable vision des faits historiques au cours de la seconde moitié du XIX ${ }^{\mathrm{e}}$ siècle. Voir D. Droixhe, L'étymon des dieux. Mythologie gauloise, archéologie et linguistique à l'âge classique, Genève, 2002.

14. Sur le «moment 1900 » en histoire de l'art comme dans les autres disciplines, histoire de la littérature, philosophie, histoire, en particulier, voir L. JENNY, La fin de l'intériorité. Théorie de l'expression et invention de l'esthétique dans les avant-gardes françaises (1885-1935), Paris, 2002 ; G. PHILIPPE, Sujet, verbe, complément. Le moment grammatical de la littérature française, 1890-1940, Paris, 2002 ; F. WORMS, Le moment 1900 en philosophie, Presses universitaires du Septentrion, 2004.

15. E. MÂLE, L'art religieux du XIII ${ }^{e}$ siècle en France. Étude sur l'iconographie du Moyen Âge et sur ses sources d'inspiration, Paris, 1898 (12éd., Paris, 1987) ; L'art religieux de la fin du Moyen Âge en France. Étude sur l'iconographie du Moyen Âge et sur ses sources d'inspiration, Paris, 1908 (7éd., Paris, 1995) ; L'art religieux du XII siècle en France. Étude sur les origines de l'iconographie du Moyen Âge, Paris, 1922 (8éd., Paris, 1998) ; L'art religieux après le concile de Trente. Étude sur l'iconographie de la fin du XVI ${ }^{e}$ siècle, du XVII ${ }^{e}$, du XVIII siècle. Italie, France, Espagne, Flandres, Paris, 1932 ( $3^{\mathrm{e}}$ éd., Paris, 1972). Pour une évaluation d'ensemble de l'œuvre, Émile Mâle (1862-1954). La construction de l'œuvre: Rome et l'Italie, Rome, 2005 (Collection de l'École française de Rome, 345).

16. E. MÂLE, « Les origines de la sculpture française du Moyen Âge », La Revue de Paris, $1^{\mathrm{er}}$ septembre 1895, p. 216.

17. ID., op. cit., p. 2 (éd. 1898).

18. ID., op. cit., p. 190 (1908).

19. R. RECHT, L'objet de l'histoire de l'art, op. cit., p. 34 ; p. 37-38 : « ... Solidement établi dans les années 1920 et 1930, sous l'enseigne de la théorie de la Gestalt mais aussi de la fortune que connaissait la morphologie goethéenne, ce formalisme, même lorsqu'il est d'une grande richesse suggestive comme chez Henri Focillon, paraît aujourd'hui singulièrement daté. Sans doute parce que l'échelle de perception a changé. » Pour une approche d'ensemble sur Henri Focillon, La vie des formes. Henri Focillon et les arts, (Musée des Beaux-Arts de Lyon, INHA, 22 janvier-26 avril 2004), Paris, 2004.

20. R. RECHT, op. cit., p. 38. 
21. B. JOBERT, « Enseigner l'histoire de l'art », Encyclopaedia Universalis, Universalia, Paris, 2002, p. 200-202.

22. Par différence avec l'Allemagne, notamment, où l'histoire de l'art assuma d'emblée le rôle public d'une instance herméneutique réfléchissant aussi bien sur le statut d'une science de l'art, que sur la question de l'objet d'art. H. Dilly, Kunstgeschichte als Institution, Francfort, 1979.

23. R. RECHT, L'objet de l'histoire de l'art, op. cit., p. 26 : «... Dans l'espace de l'histoire de l'art, l'œuvre singulière devient l'objet d'un discours et accède par conséquent à une existence non plus seulement sensible, mais intellectuelle. »

24. Georges de la Tour (Paris, mai-septembre 1972), Paris, 1972, p. 27.

25. J. THUILLIER, Théorie générale de l'histoire de l'art, Paris, 2003, p. 63 ; p. 67 : « ... Du coup, l'œuvre d'art exclut tout codage ; ou du moins, elle n'est jamais épuisée par un codage quelconque. La polysémie est sa nature même. Et devant ce signifiant qui se signifie, la part de l'interprétation individuelle est évidemment très grande. »

26. H. Belting, Das Ende der Kunstgeschichte ?, op. cit., trad. fr., p. 26.

27. A. RIEGL, Die Spätromische Kunst Industrie nach den Funden in Oesterreich-Ungarn, Vienne, 1901-1902, (trad. it., Turin, 1966), pour l'abandon de tout système normatif des valeurs et de la distinction entre le grand art et les arts mineurs. Sur son œuvre, M. OLIN, Forms of Representation in Alois Riegl's Theory of Art, The Pennsylvania State University Press, 1992.

28. R. RECHT, op. cit., p. 39-40.

29. D. Russo, « Le nom de l'artiste, entre appartenance au groupe et écriture personnelle ", L'individu au Moyen Âge, B. M. BEDos-REZAK, D. IOGNA-PRAT, Paris, 2005, p. 235-246.

30. Sur les catégories de style dans les arts visuels, reprises des genres du discours rhétorique, R. RECHT, op. cit., p. 40-41, et ID., Le croire et le voir, op. cit., p. 299-307. Pour l'exemple des sculpteurs allemands, P.-C. CLAUSSEN, « Zentrum, Peripherie, Transperipherie. Überlegungen zum Erfolg des gotischen Figurenportals an den Beispielen Chartres, Sangüesa, Magdeburg, Bamberg und den Westportalen des Domes S. Lorenzo in Genua », Studien zur Geschichte der Europäischen Skulptur im 12/13. Jahrhundert, 1994, p. 671. La question est examinée par J. WIRTH, La datation de la sculpture médiévale, Genève, 2004, p. 92, p. 194-195.

31. R. RECHT, L'objet de l'histoire de l'art, op. cit., p. 42.

32. R.-A. GoldTHWAite, Wealth and the Demand for Art in Italy, 1300-1600, The John Hopkins University Press, 1993, p. 1-9, se fondant, entre autres, sur les analyses de D. MILLER, Material Culture and Mass Consumption, Oxford, 1987. Selon ces approches, voir l'étude d'une production artistique dans un espace régional circonscrit à l'Europe du nordouest par S. CASSAGNES, D'art et d'argent. Les artistes et leurs clients dans l'Europe du Nord (XIV ${ }^{e}-X V^{e}$ siècle), Presses universitaires de Rennes, 2001.

33. R.-A. GolDTHWAITE, op. cit., p. 129-148, pour la définition et l'analyse d'une « culture matérielle » et des modes de consommation dans l'Église. Sur de nouvelles modes et de nouveaux objets au cours $\mathrm{du} \mathrm{Xv}^{\mathrm{e}}$ siècle, de nouvelles manières de les appréhender dans le lexique utilisé, ID., The Building of Renaissance Florence. An Economic and Social History, The John Hopkins University Press, 1980, pour le palais seigneurial urbain, comme nouveau type de construction pour les familles du patriciat florentin au $\mathrm{XV}^{\mathrm{e}}$ siècle ; M. BAXANDALL, The Limewood Sculptors of Renaissance Germany, New Haven, 1980, p. 24, p. 116-143, pour la sculpture allemande autour de 1500. 
34. L'artiste et le commanditaire aux derniers siècles du Moyen Âge (XIII ${ }^{e}-\mathrm{XVI}{ }^{e}$ siècle), F. JOUBERT dir., Presses de l'Université de Paris-Sorbonne, 2001, en part. p. 1-4 (EAD., « Selon la devise et portraiture... : de l'idée d'une œuvre à sa réalisation »). Sur les chantiers de construction, leur histoire économique et sociale, Du projet au chantier. Maîtres d'ouvrage et maîtres d'œuvre aux XIV ${ }^{e}-X V I^{e}$ siècles, O. СHAPELOT, Paris, 2001, en part. p. 11-33 (EAD., « Maîtrise d'ouvrage et maîtrise d'œuvre dans le bâtiment médiéval »).

35. Paris 1400. Les arts sous Charles VI, E. TABURET-DeLAhaye, Paris, 2004 ; Une fondation disparue de Jean de France, duc de Berry. La Sainte-Chapelle de Bourges, B. de CHANCELBAUdOT, Paris, 2004 ; L'art à la cour de Bourgogne. Le mécénat de Philippe le Hardi et de Jean sans Peur (1364-1419), S. Jugie et N. St. Fliegel, Paris, 2004 ; Louis d'Orléans et Valentine Visconti. Mécénat et politique autour de 1400, Blois, 2004. Sur la commande des objets précieux par les princes des fleurs de lys, E. KovAcs, L'âge d'or de l'orfêvrerie parisienne au temps des princes de Valois, Dijon, 2004. Sur la notion de « magnificence ", à partir de l'exemple médicéen, mais dans un sens de portée générale, A. D. FRASER JENKINS, "Cosimo de Medici's Patronage of Architecture and the Theory of Magnificence ", Journal of the Warburg and Courtauld Institutes 33, 1970, p. 162-170 ; G. KIPLING, The Triumph of Honour: Burgundian Origins of the Elizabethan Renaissance, Leyde, 1977, p. 3-5, sur la notion; dans les funérailles, M. GAUDE-FERRAGU, D'or et de cendres. La mort et les funérailles des princes dans le royaume de France au bas Moyen Âge, Presses universitaires du Septentrion, 2005.

36. B. Buettner, « Past Presents: New Year's Gifts at the Valois Courts ca. 1400 », The Art Bulletin 83, 2002, p. 598-625 ; EAD., « Le système des objets dans le testament de Blanche de Navarre ", Clio. Histoire, Femmes et Sociétés 19, 2004, p. 37-62, pour l'étude d'un exemple particulièrement significatif.

37. M. MADERO, Tabula Picta. La peinture et l'écriture dans le droit médiéval, Paris, 2004, pour de stimulantes analyses sur ce point.

38. D. ARASSE, «Le concept de Kunstwollen dans l'œuvre d'Aloïs Riegl », Scolies 1, 1972, p. 40 ; R. RECHT, Le croire et le voir, op. cit., p. 53-54.

39. L'emploi de wollen s'inscrivait aussi dans une tendance vitaliste caractéristique de l'époque ; W. SAUERLÄNDER, « Alois Riegl und die Enstehung der autonomen Kunstgeschichte am Fin de siècle », Fin de siècle. Zu Literatur und Kunst der Jahrhundertwende, R. BAUER, E. HeFTRICH, H. KOOFMAN, W. RASCH, W. SAUERLÄNDER et J. A. SCHMOLL dir., Francfort/M., 1987, p. 125-139 ; O. PÄCHT, Methodisches zur Kunsthistorischen Praxis, Munich, 1977, p. 147-152 (trad. fr., J. Lacoste, Paris, 1994, p. 123).

40. A. RIEGL, « Naturwerk und Kunstwerk I (1901) », réédité dans ID., Gesammelte Aufsätze, Augsbourg-Vienne, 1929, p. 51-64.

41. ID., loc. cit., p. 60-64.

42. E. WIND, «Warburg Begriff der Kulturwissenschaft und seine Bedeutung für die Ästhetik », Zeitschrift für Ästhetik und Allgemeine Kunstwissenschaft 25, 1931, p. 163-179 (repr. dans ID., The Eloquence of Symbols. Studies in Humanist Art, Oxford, 1983, p. 21-35).

43. H. ZERNER, « L'histoire de l'art d'Alois Riegl : un formalisme tactique », Critique aoûtseptembre 1975, p. 946-947 ; P. РнILIPPOT, « Présentation » de L'origine de l'art baroque à Rome d'Alois Riegl, Paris, 1993, p. 10-11.

44. E. WIND, «Zur Systematik der Künstlerischen Probleme », Zeitschrift für Ästhetik und Allgemeine Kunstwissenschaft 18, 1924, p. 438-486 ; H. SEDLMAYR, « Die Quintessenz der Lehren Riegls », dans A. RIEGL, Gesammelte Aufsätze, op. cit., p. XI-XXXIV. 
45. E. PANOFSKY, «Der Begriff des Kunstwollens », Zeitschrift für Ästhetic und Allgemeine Kunstwissenschaft 14, 1920, p. 321-339 (trad. fr. par G. Ballange dir., Paris, 1975, p. 197-221).

46. ID., Studies in Iconology, Introduction, Oxford University Press, 1939 (trad. fr. par Cl. Herbette, B. Teyssèdre, Paris, 1967).

47. N. Belmont, "Arnold van Gennep », Hier pour demain. Arts, traditions, patrimoine, J. CUISENIER dir., Paris, 1980 ; Chroniques de folklore d'Arnold van Gennep. Recueil de textes parus dans le Mercure de France, 1905-1949, J.-M. PRIVAT éd., Paris, 2001. Sur la sémantique panofskyenne, J. COURTES, « Un modèle suggestif : le motif panofskyen comme forme figurative », dans ID., Le conte populaire : poétique et mythologie, Paris, 1986, p. 21-40.

48. Sur ce tournant, E. PANOFSKY, «The History of Art as a Humanistic Discipline », dans T. M. GREENE, The Meaning of the Humanities, Princeton University Press, 1940, p. 89-119 ; repris dans ID., Meaning in the Visual Arts. The Renaissance : Artist, Scientist, Genius, Princeton University Press, 1955, p. 1-25 (trad. fr. par M. et B. Teyssèdre, L'œuvre d'art et ses significations. Essais sur les arts visuels, Paris, 1969, p. 27-52.

49. R. RECHT, L'objet de l'histoire de l'art, op. cit., p. 56-57.

\section{AUTEUR}

\section{DANIEL RUSSO}

Université de Bourgogne, UMR 5594, 2 boulevard Gabriel, F-21000 Dijon 